\title{
Rational Solutions of the H3 and Q1 Models in the ABS Lattice List*
}

Ying SHI and Da-jun ZHANG

Department of Mathematics, Shanghai University, Shanghai 200444, P.R. China

E-mail: shiying0707@shu.edu.cn,djzhang@staff.shu.edu.cn

URL: http://www.science.shu.edu.cn/siziduiwu/zdj/index.htm

Received January 31, 2011, in final form May 04, 2011; Published online May 09, 2011

doi:10.3842/SIGMA.2011.046

\begin{abstract}
In the paper we present rational solutions for the H3 and Q1 models in the Adler-Bobenko-Suris lattice list. These solutions are in Casoratian form and are generated by considering difference equation sets satisfied by the basic Casoratian column vector.
\end{abstract}

Key words: Casoratian; bilinear; rational solutions; H3; Q1

2010 Mathematics Subject Classification: 37K10

\section{Introduction}

Recently, for many discrete systems which are integrable in the sense of multi-dimensional consistency [1, 2], their explicit soliton solutions were derived via constructive approaches [3, 4, 5, 6, 7, 8, 9] such as the Cauchy Matrix approach and bilinear method. For a continuous integrable system, once we have its multi-soliton solutions, rational solutions usually can be found through limiting procedures (cf. $[10,11])$. In discrete case, limiting procedures for getting rational solutions are more delicate and they often lead to a trivialization of the Cauchy matrix. However, bilinear method has been successfully applied to get limit solutions of some multidimensionally consistent lattices. This was mentioned in [7] for the lattice Boussinesq equation and then discussed in detail in [12] for the H1 equation in the Adler-Bobenko-Suris (ABS)'s list [2]. In fact, with the help of bilinear forms and Casoratians (see [6, 7, 12]), one can transfer a lattice equation to a linear difference equation set satisfied by a $N$ th-order Casoratian column vector. Usually there will be a coefficient matrix appearing in the difference equation set. When the coefficient matrix has $N$ distinct eigenvalues one gets $N$-soliton solutions, and when it has $N$ same eigenvalues one gets limit solutions.

In this paper, we will see that H3 and Q1 models in the ABS's list [2] admit rational solutions. In [6] Casoratian solutions for H3 and Q1 have been given with explicit Casoratian entries. In this paper, we will first replace these explicit entries by the difference equation sets satisfied by them, by which it is possible to get more solutions beyond solitons. We need to introduce a coefficient matrix for each difference equation set. Then, by discussing eigenvalues of the coefficient matrix we can get several "generalized" solutions, and one of which is rational type.

The paper is organized as follows. Section 2 provides basic notations for lattice variables, Casoratians and so forth. In Section 3 we derive rational solutions for H3 and in Section 4 for Q1.

\footnotetext{
${ }^{\star}$ This paper is a contribution to the Proceedings of the Conference "Integrable Systems and Geometry" (August 12-17, 2010, Pondicherry University, Puducherry, India). The full collection is available at http://www.emis.de/journals/SIGMA/ISG2010.html
} 


\section{Preliminary}

Let us introduce some notations. Given a base point $u_{n, m}=u$, we indicate the shifts in $n, m$ direction by up/down-tilde and up/down-hat, say

$$
\widetilde{u}=u_{n+1, m}, \quad \widehat{u}=u_{n, m+1}, \quad \underset{\sim}{u}=u_{n-1, m}, \quad \underset{\wedge}{u}=u_{n, m-1} .
$$

With these notations the lattice equations of our interest are

$$
\begin{aligned}
& \mathrm{H} 3 \equiv p(u \widetilde{u}+\widehat{u} \widehat{\widetilde{u}})-q(u \widehat{u}+\widetilde{u} \widehat{\widetilde{u}})+\delta\left(p^{2}-q^{2}\right)=0, \\
& \mathrm{Q} 1 \equiv p(u-\widehat{u})(\widetilde{u}-\widehat{\widetilde{u}})-q(u-\widetilde{u})(\widehat{u}-\widehat{\widetilde{u}})+\delta^{2} p q(p-q)=0,
\end{aligned}
$$

where $p$ and $q$ are independent parameters of $n$ and $m$ respectively, and $\delta$ is an arbitrary constants. For generating soliton solutions, one needs to introduce an auxiliary direction $l$ which provides parameters of the solitons by Bäcklund transformation constructed from multidimensional consistency. Shifts in $l$ direction are indicated by up/down-bar as follows, $\bar{u}=$ $u(l+1), \underline{u}=u(l-1)$.

Casoratian is a discrete version of Wronskian. It is a determinant of a Casorati matrix composed of a basic column vector, i.e.,

$$
\left|\psi\left(n, m, l_{1}\right), \psi\left(n, m, l_{2}\right), \ldots, \psi\left(n, m, l_{N}\right)\right|=\left|l_{1}, l_{2}, \ldots, l_{N}\right|,
$$

where the basic column vector is

$$
\psi(n, m, l)=\left(\psi_{1}(n, m, l), \psi_{2}(n, m, l), \ldots, \psi_{N}(n, m, l)\right)^{T} .
$$

Following the standard shorthand notation given in [13], some $N$ th-order Casoratians that we often use are indicated by

$$
\begin{aligned}
& |\widehat{N-1}|=|0,1, \ldots, N-1|, \quad|\widehat{N-2}, N|=|0,1, \ldots, N-2, N|, \\
& |-1, \widehat{N-1}|=|-1,1,2, \ldots, N-1| .
\end{aligned}
$$

The above Casoratians are defined in terms of the shifts in $l$ direction. In fact, we can organize columns in terms of the shifts of $n$ or $m$. By the operators $E^{\nu}(\nu=1,2,3)$ we denote the operations

$$
E^{1} \psi=\widetilde{\psi}, \quad E^{2} \psi=\widehat{\psi}, \quad E^{3} \psi=\bar{\psi},
$$

then we can define a Casoratian w.r.t. $E^{\nu}$-shift,

$$
|\widehat{N-1}|_{[\nu]}=\left|\psi, E^{\nu} \psi,\left(E^{\nu}\right)^{2} \psi, \ldots,\left(E^{\nu}\right)^{N-1} \psi\right|, \quad \nu=1,2,3
$$

We note that if the column vector $\psi$ satisfies

$$
\bar{\psi}=\widetilde{\psi}+\alpha \psi \quad \text { or } \quad \bar{\psi}=\widehat{\psi}+\beta \psi,
$$

where $\alpha$ and $\beta$ are some constant, then we have

$$
|\widehat{N-1}|_{[3]}=|\widehat{N-1}|_{[1]} \quad \text { or } \quad|\widehat{N-1}|_{[3]}=|\widehat{N-1}|_{[2]} .
$$

In addition to the above notations and properties, we need the the following Laplace expansion identity for Casoratian verification.

Lemma 1 ([13]). Suppose that $\mathbf{B}$ is a $N \times(N-2)$ matrix, and $\boldsymbol{a}, \boldsymbol{b}, \boldsymbol{c}, \boldsymbol{d}$ are $N$ th-order column vectors, then

$$
|\mathbf{B}, \mathbf{a}, \mathbf{b} \| \mathbf{B}, \mathbf{c}, \mathbf{d}|-|\mathbf{B}, \mathbf{a}, \mathbf{c}||\mathbf{B}, \mathbf{b}, \mathbf{d}|+|\mathbf{B}, \mathbf{a}, \mathbf{d}||\mathbf{B}, \mathbf{b}, \mathbf{c}|=0 .
$$




\section{Rational solutions for H3}

There are two different bilinear forms related to the H3 equation (2.1), which are [6]

$$
\begin{aligned}
& \mathcal{B}_{1} \equiv 2 c f \widetilde{f}+(a-c) \widetilde{\bar{f}} \underline{f}-(a+c) \bar{f} \underline{\tilde{f}}=0, \\
& \mathcal{B}_{2} \equiv 2 c f \widehat{f}+(b-c) \hat{\bar{f}} \underline{f}-(b+c) \bar{f} \underline{\widehat{f}}=0,
\end{aligned}
$$

and

$$
\begin{aligned}
& \mathcal{B}_{1}^{\prime} \equiv(b+c) \widehat{\widetilde{f}} \bar{f}+(a-c) f \widehat{\widetilde{\bar{f}}}-(a+b) \widetilde{\bar{f}} \hat{\bar{f}}=0, \\
& \mathcal{B}_{2}^{\prime} \equiv(c-b) \hat{\widetilde{\widetilde{f}}} \underline{f}-(a+c) \hat{f} \underline{\widetilde{\widetilde{f}}}+(a+b) \tilde{\widetilde{f}} \underline{\widehat{f}}=0, \\
& \mathcal{B}_{3}^{\prime} \equiv(c-a)(b+c) \widetilde{\widetilde{f}} \underline{\hat{f}}+(a+c)(b-c) \hat{\bar{f}} \underline{\tilde{f}}+2 c(a-b) f \widehat{\widetilde{f}}=0 \text {, }
\end{aligned}
$$

via the same transformation

$$
u=A \alpha^{n} \beta^{m} \frac{\bar{f}}{f}+B \alpha^{-n} \beta^{-m} \frac{f}{f}, \quad A B=-\frac{1}{4} r \delta,
$$

where the parametrization is

$$
\frac{r^{2} c^{2}}{c^{2}-a^{2}}=p^{2}, \quad \frac{r^{2} c^{2}}{c^{2}-b^{2}}=q^{2}, \quad \alpha^{2}=-\frac{a-c}{a+c}, \quad \beta^{2}=-\frac{b-c}{b+c} .
$$

Both bilinear forms can be solved by $[6]$

$$
f(\psi)=|\widehat{N-1}|_{[3]}
$$

with the Casoratian column vector $\psi$ (see the structure (2.3)) composed of

$$
\psi_{i}(n, m, l)=\rho_{i}^{+}\left(a+k_{i}\right)^{n}\left(b+k_{i}\right)^{m}\left(c+k_{i}\right)^{l}+\rho_{i}^{-}\left(a-k_{i}\right)^{n}\left(b-k_{i}\right)^{m}\left(c-k_{i}\right)^{l},
$$

where $\rho_{i}^{ \pm}$and $k_{i}$ are parameters.

Now we consider a generalization of the above $\psi$. We will discuss the linear difference equation set satisfied by $\psi$. First, there should exist symmetric relationship between $(n, a)$ pair and $(m, b)$ pair. This can be seen from the symmetric position of $n$ and $m$ appearing in the H3 equation. In addition, from the bilinear forms (3.1) and (3.2) this symmetric relationship can be extended to include $(l, c)$ pair. Since $\psi$ plays a role of the basic Casoratian column vector, it will consequently keep the $n-m-l$ symmetric property.

In the generalization procedure, Toeplitz matrices play important roles. A $N$ th-order lower triangular Toeplitz matrix (LTTM) $\mathcal{F}$ is a matrix of the form (see [11] for more properties)

$$
\mathcal{F}=\left(\begin{array}{cccccc}
f_{0} & 0 & 0 & \cdots & 0 & 0 \\
f_{1} & f_{0} & 0 & \cdots & 0 & 0 \\
f_{2} & f_{1} & f_{0} & \cdots & 0 & 0 \\
\cdots & \cdots & \cdots & \cdots & \cdots & \cdots \\
f_{N-1} & f_{N-2} & f_{N-3} & \cdots & f_{1} & f_{0}
\end{array}\right), \quad f_{j} \in \mathbb{C} .
$$

$\mathcal{F}(k)$ is called a $N$ th-order LTTM generated from the function $F(k)$, if in (3.6)

$$
f_{j}=\frac{1}{j !} \partial_{k}^{j} F(k),
$$

where the 'seed' function $F(k)$ is arbitrarily differentiable w.r.t. $k$. 
Lemma 2. Let non-zero functions $F(k, l)$ and $G(k)$ be sufficiently smooth w.r.t. $k$ and satisfy

$$
\partial_{l} F(k, l)=F(k, l) G(k) .
$$

Here $l$ is an auxiliary argument of $F(k, l)$. If $\mathcal{F}(k, l)$ and $\mathcal{G}(l)$ are respectively two $N$ th-order LTTMs generated from $F(k, l)$ and $G(k)$, then we have

$$
\partial_{l} \mathcal{F}(k, l)=\mathcal{F}(k, l) \mathcal{G}(k) .
$$

\section{Proof.}

$$
\begin{aligned}
\partial_{l} f_{j} & =\frac{1}{j !} \partial_{k}^{j} \partial_{l} F(k, l)=\frac{1}{j !} \partial_{k}^{j}(F(k, l) G(k))=\frac{1}{j !} \sum_{s=0}^{j} C_{j}^{s}\left(\partial_{k}^{j-s} F(k, l)\right)\left(\partial_{k}^{s} G(k)\right) \\
& =\sum_{s=0}^{j}\left(\frac{1}{(j-s) !} \partial_{k}^{j-s} F(k, l)\right)\left(\frac{1}{s !} \partial_{k}^{s} G(k)\right)=\sum_{s=0}^{j} f_{j-s} \cdot g_{s} .
\end{aligned}
$$

This yields the relation (3.7).

Corollary 1. Under the condition of Lemma 2, the product of $\operatorname{LTTMs} \mathcal{F}(k, l) \mathcal{F}^{-1}(k, l+1)$ is independent of $l$.

Proof. From (3.7) we have

$$
\mathcal{F}^{-1}(k, l) \cdot \partial_{l} \mathcal{F}(k, l)=\mathcal{G}(k),
$$

which means $\mathcal{F}^{-1}(k, l) \cdot \partial_{l} \mathcal{F}(k, l)$ is independent of $l$, i.e.,

$$
\mathcal{F}^{-1}(k, l) \cdot \partial_{l} \mathcal{F}(k, l)=\mathcal{F}^{-1}(k, l+1) \cdot \partial_{l} \mathcal{F}(k, l+1) .
$$

Noting that

$$
\begin{aligned}
& \partial_{l}\left(\mathcal{F}(k, l) \mathcal{F}^{-1}(k, l+1)\right) \\
& \quad=\left(\partial_{l} \mathcal{F}(k, l)\right) \mathcal{F}^{-1}(k, l+1)-\mathcal{F}(k, l) \mathcal{F}^{-1}(k, l+1)\left(\partial_{l} \mathcal{F}(k, l+1)\right) \mathcal{F}^{-1}(k, l+1)
\end{aligned}
$$

and then replacing $\mathcal{F}^{-1}(k, l+1) \cdot \partial_{l} \mathcal{F}(k, l+1)$ by $\mathcal{F}^{-1}(k, l) \cdot \partial_{l} \mathcal{F}(k, l)$ yield

$$
\partial_{l}\left(\mathcal{F}(l) \mathcal{F}^{-1}(l+1)\right)=0 .
$$

Thus we complete the proof.

Now let us come to the main results of $\mathrm{H} 3$.

Theorem 1. For H3 the Casoratian $f(\psi)$ defined in (3.4) solves the bilinear forms (3.1) and (3.2) if $\psi$ satisfies

$$
\bar{\psi}=\widetilde{\psi}+(c-a) \psi,
$$

and there is a $N$ th-order auxiliary vector $\sigma(n, m, l)$ such that

$$
\psi=A_{[l]} \sigma,
$$

and

$$
\underline{\sigma}=-\widehat{\sigma}+(b+c) \sigma,
$$

where $A_{[l]}$ is a Nth-order transform matrix, $A_{[l]} A_{[l+1]}^{-1}$ is independent of $l$, and the subscript $[l]$ specially means that $A_{[l]}$ only depends on $l$ but is independent of $(n, m)$. Moreover, in the light of $n$-m-l symmetric property and (3.8b), the following relation

$$
\psi=A_{[n]} \omega, \quad \psi=A_{[m]} \phi
$$

automatically holds, where $\omega(n, m, l)$ and $\phi(n, m, l)$ are auxiliary vectors. 
Proof. The proof is similar to the one with concrete $\psi_{i}(3.5)$ in [6], but here we need to carefully examine the role played by the transform matrix $A_{[l]}$. We only take (3.1a) as an example. By a down-tilde-shift it goes to

$$
2 c \underset{\sim}{f} f+(a-c) \bar{f} \underset{\sim}{f}-(a+c) \underset{\sim}{f} \underline{f}=0 .
$$

The shift relation (3.8a) and its symmetric forms suggest

$$
f(\psi)=|\widehat{N-1}|_{[i]}=|\widehat{N-1}|_{[j]}, \quad i, j=1,2,3 .
$$

So for (3.9) we consider $f(\psi)=|\widehat{N-1}|_{[2]}$ for convenience. Same as the derivation in [6], we have

$$
-(a-b)^{N-2} \underset{\sim}{f}(\psi)=|\widehat{N-2}, \underset{\sim}{\psi}(N-2)|_{[2]}, \quad-(c-b)^{N-2} \underline{f}(\psi)=|\widehat{N-2}, \underline{\psi}(N-2)|_{[2]},
$$

and

$$
(a-c)(c-b)^{N-2}(a-b)^{N-2} \underset{\sim}{\sim}(\psi)=|\widehat{N-3}, \underline{\psi}(N-2), \underset{\sim}{\psi}(N-2)|_{[2]} .
$$

Besides, with the help of the shift relation (3.8c) for $\sigma$, we have

$$
(c+b)^{N-2} \bar{f}(\sigma)=|\sigma(0), \sigma(1), \ldots, \sigma(N-2), \bar{\sigma}(N-2)|_{[2]} .
$$

Then, noting that $f(\psi)=\left|A_{[l]}\right| f(\sigma)$ in terms of $m$-shift construction of Casoratians and defining

$$
\stackrel{\circ}{E}^{3} \psi=A_{[l]} A_{[l+1]}^{-1} E^{3} \psi
$$

we find from (3.10) that

$$
(c+b)^{N-2} \bar{f}(\psi)=\left|A_{[l+1]}\right|\left|A_{[l]}^{-1}\right|\left|\widehat{N-2}, \stackrel{\circ}{E}^{3} \psi(N-2)\right|_{[2]},
$$

and further

$$
(a+c)(c+b)^{N-2}(a-b)^{N-2} \underset{\sim}{f}(\psi)=\left|A_{[l+1]}\right|\left|A_{[l]}^{-1}\right|\left|\widehat{N-3}, \underset{\sim}{\sim}(N-2), \stackrel{\circ}{E}^{3} \psi(N-2)\right|_{[2]} .
$$

Imposing a down-bar shift on (3.11) and using the relation (3.8a) yield

$$
2 c(c-b)^{N-2}(c+b)^{N-2} f(\psi)=\left|A_{[l+1]}\right|\left|A_{[l]}^{-1}\right|\left|\widehat{N-3}, \underline{\psi}(N-2), \stackrel{\circ}{E}^{3} \psi(N-2)\right|_{[2]},
$$

where we have made use of the assumption of $A_{[l]} A_{[l+1]}^{-1}$ being independent of $l$. Now collecting these obtained formulae, substituting them into the l.h.s. of (3.9) and using Lemma 1, we can prove (3.9). The proof of other formulae in the bilinear forms (3.1) and (3.2) are similar and we do not give further details.

Now let us see what is new the theorem brings. One choice satisfying Theorem 1 is to take $\psi$ which is composed of $(3.5), \sigma$ composed of

$$
\sigma_{i}(n, m, l)=\rho_{i}^{+}\left(a+k_{i}\right)^{n}\left(b+k_{i}\right)^{m}\left(c-k_{i}\right)^{-l}+\rho_{i}^{-}\left(a-k_{i}\right)^{n}\left(b-k_{i}\right)^{m}\left(c+k_{i}\right)^{-l},
$$

and

$$
A_{[l]}=\operatorname{Diag}\left(A\left(k_{1}, l\right), A\left(k_{2}, l\right), \ldots, A\left(k_{N}, l\right)\right), \quad A\left(k_{j}, l\right)=\left(c^{2}-k_{j}^{2}\right)^{l} .
$$

This is nothing but the result given in [6] and it generates $N$-soliton solutions for H3. 
Now let $A_{[l]}$ be a $N$ th-order LTTM in the form of (3.6), which is generated from $A\left(k_{1}, l\right)=$ $\left(c^{2}-k_{1}^{2}\right)^{l}$, i.e.,

$$
a_{j}=\frac{1}{j !} \partial_{k_{1}}^{j}\left(c^{2}-k_{1}^{2}\right)^{l} .
$$

Then, the desirable Casoratian column vector $\psi$ can be taken as

$$
\psi(n, m, l)=\mathcal{A}_{+} \psi^{+}(n, m, l)+\mathcal{A}_{-} \psi^{-}(n, m, l),
$$

where

$$
\begin{aligned}
& \psi^{ \pm}(n, m, l)=\left(\psi_{1}^{ \pm}(n, m, l), \psi_{2}^{ \pm}(n, m, l), \ldots, \psi_{N}^{ \pm}(n, m, l)\right)^{T}, \\
& \psi_{i}^{ \pm}(n, m, l)=\frac{1}{(i-1) !} \partial_{k_{1}}^{i-1}\left[\rho_{1}^{ \pm}\left(a \pm k_{1}\right)^{n}\left(b \pm k_{1}\right)^{m}\left(c \pm k_{1}\right)^{l}\right],
\end{aligned}
$$

and $\mathcal{A}_{ \pm}$are two arbitrary non-singular LTTMs. The corresponding auxiliary vector $\sigma$ is given by

$$
\sigma=\mathcal{A}_{+} \sigma^{+}(n, m, l)+\mathcal{A}_{-} \sigma^{-}(n, m, l)
$$

where

$$
\begin{aligned}
& \sigma^{ \pm}(n, m, l)=\left(\sigma_{1}^{ \pm}(n, m, l), \sigma_{2}^{ \pm}(n, m, l), \ldots, \sigma_{N}^{ \pm}(n, m, l)\right)^{T} \\
& \sigma_{i}^{ \pm}(n, m, l)=\frac{1}{(i-1) !} \partial_{k_{1}}^{i-1}\left[\rho_{1}^{ \pm}\left(a \pm k_{1}\right)^{n}\left(b \pm k_{1}\right)^{m}\left(c \mp k_{1}\right)^{l}\right] .
\end{aligned}
$$

We note that in the light of Corollary $1, A_{[l]} A_{[l+1]}^{-1}$ is independent of $l$. Besides, to avoid the generation of zero by high order derivatives we may let $l \geq N$ or $l \notin \mathbb{Z}^{+}$. (This can be done, for example, by taking $\left(c \pm k_{1}\right)^{l_{0}} \rho_{1}^{ \pm}$in place of the original $\rho_{1}^{ \pm}$where $l_{0}$ is either a positive integer greater than $N-1$ or not a positive integer. As an example, see (3.13d) where we have taken $l_{0}=2$.)

As the simplest nontrivial case we take $N=2$ and thus the Casoratian $f$ is in the form of

$$
f=|\psi(n, m, 0), \psi(n, m, 1)|_{[3]}
$$

where

$$
\begin{aligned}
& \psi(n, m, l)=\left(\psi_{1}(n, m, l), \partial_{k_{1}} \psi_{1}(n, m, l)\right)^{T}, \\
& \psi_{1}(n, m, l)=\psi_{1}^{+}(n, m, l)+\psi_{1}^{-}(n, m, l), \\
& \psi_{1}^{ \pm}(n, m, l)=\rho_{1}^{ \pm}\left(a \pm k_{1}\right)^{n}\left(b \pm k_{1}\right)^{m}\left(c \pm k_{1}\right)^{2+l},
\end{aligned}
$$

and we have taken $\mathcal{A}_{ \pm}=I$. In this case, the coefficient (or transform) matrix $A_{[l]}$ is a 2nd-order LTTM generated from $A\left(k_{1}, l\right)=\left(c^{2}-k_{1}^{2}\right)^{2+l}$, i.e.,

$$
A_{[l]}=\left|\begin{array}{cc}
\left(c^{2}-k_{1}^{2}\right)^{2+l} & 0 \\
\partial_{k_{1}}\left(c^{2}-k_{1}^{2}\right)^{2+l} & \left(c^{2}-k_{1}^{2}\right)^{2+l}
\end{array}\right| .
$$

Then, substituting (3.13a) into the transformation (3.3) yields a solution to H3, which is different from 2-soliton solution,

$$
\begin{aligned}
& u_{n, m}=A \alpha^{n} \beta^{m} \frac{\left(c-k_{1}\right)^{2}-\left[D_{1}+4 k_{1} c\left(c^{2}-k_{1}^{2}\right)^{-1}\right]\left(c^{2}-k_{1}^{2}\right) Q_{n, m}-\left(c+k_{1}\right)^{2} Q_{n, m}^{2}}{1-D_{1} Q_{n, m}-Q_{n, m}^{2}} \\
& +B \alpha^{-n} \beta^{-m} \frac{\left(c-k_{1}\right)^{-2}-\left[D_{1}-4 k_{1} c\left(c^{2}-k_{1}^{2}\right)^{-1}\right]\left(c^{2}-k_{1}^{2}\right)^{-1} Q_{n, m}-\left(c+k_{1}\right)^{-2} Q_{n, m}^{2}}{1-D_{1} Q_{n, m}-Q_{n, m}^{2}}
\end{aligned}
$$


where

$$
\begin{aligned}
& Q_{n, m}=\left(\frac{c+k_{1}}{c-k_{1}}\right)^{2}\left(\frac{a+k_{1}}{a-k_{1}}\right)^{n}\left(\frac{b+k_{1}}{b-k_{1}}\right)^{m} \rho_{0,0}, \\
& D_{1}=\frac{4 k_{1}\left[a\left(b^{2}-k_{1}^{2}\right)\left(c^{2}-k_{1}^{2}\right) n+b\left(a^{2}-k_{1}^{2}\right)\left(c^{2}-k_{1}^{2}\right) m+2 c\left(a^{2}-k_{1}^{2}\right)\left(b^{2}-k_{1}^{2}\right)\right]}{\left(a^{2}-k_{1}^{2}\right)\left(b^{2}-k_{1}^{2}\right)\left(c^{2}-k_{1}^{2}\right)} \\
& A B=-\frac{1}{4} r \delta,
\end{aligned}
$$

and the constants $\rho_{1}^{ \pm}$have been absorbed into the parameter $\rho_{0,0}$. Comparing with the previous results in $[5,6]$, the function $D_{1}$ which depends on $n, m$ is new, and this is the generalization.

Recalling the transform(or coefficient) matrix $A_{[l]}$ defined in (3.12), which is a diagonal and has $N$ distinct eigenvalues. It leads to $N$-soliton solutions. When $A_{[l]}$ is the $N$ th-order LTTM generated from $A\left(k_{1}, l\right)=\left(c^{2}-k_{1}^{2}\right)^{l}$, which is a matrix with $N$ same eigenvalues $A\left(k_{1}, l\right)$, we get a kind of solutions which is closely related to some limiting procedures (see $[11,12])$ and different from solitons. Such solutions we call limit solutions. Usually taking $k_{1}=0$ in such limit solutions one may get rational solutions. In practice, we may take $\psi_{1}$ to be an even function of $k_{1}$, for example,

$$
\psi_{1}(n, m, l)=\left(a+k_{1}\right)^{n}\left(b+k_{1}\right)^{m}\left(c+k_{1}\right)^{l_{0}+l}+\left(a-k_{1}\right)^{n}\left(b-k_{1}\right)^{m}\left(c-k_{1}\right)^{l_{0}+l},
$$

where $l_{0}$ is some constant as we mentioned before. Correspondingly, a nontrivial solution of (3.8a) can be taken as

$$
\psi(n, m, l)=\left(\psi_{1}(n, m, l), \frac{1}{2 !} \partial_{k_{1}}^{2} \psi_{1}(n, m, l), \ldots, \frac{1}{(2 N-2) !} \partial_{k_{1}}^{2 N-2} \psi_{1}(n, m, l)\right)^{T},
$$

and the transform matrix $A_{[l]}$ is a LTTM in the form of (3.6) with

$$
a_{j}=\frac{1}{(2 j) !} \partial_{k_{1}}^{2 j}\left(c^{2}-k_{1}^{2}\right)^{l_{0}+l}, \quad j=0,1, \ldots, N-1 .
$$

Then, the Casoratian for rational solutions is

$$
f=|\psi(n, m, 0), \psi(n, m, 1), \ldots, \psi(n, m, N-1)|_{k_{1}=0} .
$$

The following is a rational solution (with $N=2$ and $l_{0}=2$ ),

$$
u=A \alpha^{n} \beta^{m} c^{2}\left(1+\frac{a b}{c(b n+a m)+2 a b}\right)+B \alpha^{-n} \beta^{-m} c^{-2}\left(1-\frac{a b}{c(b n+a m)+2 a b}\right),
$$

where $A B=-\frac{1}{4} r \delta$.

\section{Rational solutions for Q1}

\subsection{For bilinearization-I}

There are two types of bilinear forms for Q1 equation (2.2). One is [6]

$$
\begin{aligned}
& \mathcal{Q}_{1} \equiv \widehat{\widetilde{\widetilde{f}}} f(b-\delta)+\widetilde{\widetilde{f}} f(a+\delta)-\widetilde{\widetilde{f}} \widehat{f}(a+b)=0, \\
& \mathcal{Q}_{2} \equiv \widehat{\widetilde{\bar{f}}} f(a-b)+\widetilde{\bar{f}} \widehat{f}(b+\delta)-\widetilde{\bar{f} f}(a+\delta)=0, \\
& \mathcal{Q}_{3} \equiv-\widetilde{\bar{f}} \widehat{f}+\widetilde{\bar{f}} \widehat{\mathfrak{g}}(-a+\delta)+\widetilde{\widetilde{f}} \widehat{\bar{f}}+\widehat{\bar{f}} \widetilde{\mathfrak{g}}(b-\delta)+\widetilde{f} \widehat{\widetilde{g}}(a-b)=0, \\
& \mathcal{Q}_{4}=\widehat{\widetilde{\widetilde{f}}} \mathfrak{g}(a-b)+\widetilde{\bar{f}} \widehat{\mathfrak{g}}(a+b)-\widehat{\bar{f}} \widetilde{\mathfrak{g}}(a+b)+\widetilde{f} \widehat{\widetilde{\mathfrak{g}}}(-a+b)=0,
\end{aligned}
$$


where the transformation is

$$
u=\alpha n+\beta m+\gamma-\left(c^{2} / r-\delta^{2} r\right) \frac{\mathfrak{g}}{f}
$$

with parametrization

$$
\alpha=p a, \quad \beta=q b, \quad \frac{c^{2} / r-\delta^{2} r}{a^{2}-\delta^{2}}=p, \quad \frac{c^{2} / r-\delta^{2} r}{b^{2}-\delta^{2}}=q .
$$

By examining the Casoratian verification we find solutions of Q1 admit the following generalization.

Theorem 2. The bilinear Q1 (4.1) can be solved by

$$
f(\psi)=|\widehat{N-1}|_{[3]}, \quad \mathfrak{g}(\psi)=|-1, \widehat{N-1}|_{[3]},
$$

if $\psi$ satisfies the shift relation

$$
\bar{\psi}=\widetilde{\psi}+(\delta-a) \psi
$$

as well as $n$-m-l-symmetric property, and there are $N$ th-order auxiliary vector $\sigma(n, m, l)$ and $\phi(n, m, l)$ such that

$$
\psi=A_{[l]} \sigma, \quad \bar{\sigma}=-\underset{\wedge}{\sigma}+(b+\delta) \sigma,
$$

and

$$
\psi=A_{[n]} A_{[m]} \phi, \quad \bar{\phi}=-\underset{\sim}{\phi}+(a+\delta) \phi, \quad \bar{\phi}=-\underset{\sim}{\phi}+(b+\delta) \phi,
$$

where $A_{[l]}$ is defined as in Theorem $1, A_{[l]} A_{[l+1]}^{-1}$ is independent of $l, A_{[n]}$ and $A_{[m]}$ posses properties similar to $A_{[l]}$.

The proof is similar to the one in the previous section for H3 and in [6] for Q1. We skip it. $N$-soliton solutions [6] can be derived from those Casoratians by taking the transform matrixes as

$$
A_{[\mu]}=\operatorname{Diag}\left(A\left(k_{1}, \mu\right), A\left(k_{2}, \mu\right), \ldots, A\left(k_{N}, \mu\right)\right), \quad A\left(k_{j}, \mu\right)=\left(x_{\mu}^{2}-k_{j}^{2}\right)^{\mu},
$$

where

$$
\mu=n, m, l, \quad x_{1}=a, \quad x_{2}=b, \quad x_{3}=\delta,
$$

and the basic column vector $\psi$ composed of

$$
\psi_{i}(n, m, l)=\rho_{i}^{+}\left(a+k_{i}\right)^{n}\left(b+k_{i}\right)^{m}\left(\delta+k_{i}\right)^{l}+\rho_{i}^{-}\left(a-k_{i}\right)^{n}\left(b-k_{i}\right)^{m}\left(\delta-k_{i}\right)^{l} .
$$

As a generalization, $A_{[\mu]}$ can be a LTTM generated from $A\left(k_{1}, \mu\right)$. In this case, $\psi$ can be taken as

$$
\psi(n, m, l)=\mathcal{A}_{+} \psi^{+}(n, m, l)+\mathcal{A}_{-} \psi^{-}(n, m, l),
$$

with

$$
\begin{aligned}
& \psi^{ \pm}(n, m, l)=\left(\psi_{1}^{ \pm}(n, m, l), \psi_{2}^{ \pm}(n, m, l), \ldots, \psi_{N}^{ \pm}(n, m, l)\right)^{T}, \\
& \psi_{i}^{ \pm}(n, m, l)=\frac{1}{(i-1) !} \partial_{k_{1}}^{i-1}\left[\rho_{1}^{ \pm}\left(a \pm k_{1}\right)^{n}\left(b \pm k_{1}\right)^{m}\left(\delta \pm k_{1}\right)^{l_{0}+l}\right],
\end{aligned}
$$


and arbitrary $N$ th-order LTTMs $\mathcal{A}_{ \pm}$. Here we add the parameter $l_{0}$ which, as before, plays a role of avoiding triviality of some high order derivatives. Rational solutions may come out by taking $k_{1}=0$ in the above $\psi(n, m, l)$.

As examples we give two solutions which are not soliton solutions. The first one is

$$
u_{n, m}=\alpha n+\beta m+\gamma+2\left(c^{2} / r-\delta^{2} r\right) \frac{\left(\delta+k_{1}\right)-\left[\delta E_{1}-2 k_{1}\left(\delta^{4}-k_{1}^{4}\right)\right] Q_{n, m}-\left(\delta-k_{1}\right) Q_{n, m}^{2}}{\left(\delta^{2}-k_{1}^{2}\right)\left(1-E_{1} Q_{n, m}-Q_{n, m}^{2}\right)},
$$

where $Q_{n, m}$ and $E_{1}$ are given in (3.14b) and (3.14c) with $\delta$ in place of $c$. This solution is derived from the transformation (4.2) in which we use the 2nd-order Casoratians

$$
f=|\psi(n, m, 0), \psi(n, m, 1)|, \quad g=|\psi(n, m,-1), \psi(n, m, 1)|
$$

with column vector (4.4) where $\mathcal{A}_{ \pm}=I$ and $l_{0}=2$. Another example is a rational solution,

$$
u_{n, m}=\alpha n+\beta m+\gamma-\delta^{-1}\left(c^{2} / r-\delta^{2} r\right)\left(2-\frac{a b}{\delta(b n+a m)+2 a b}\right) .
$$

This is derived from the 2nd-order Casoratians

$$
f=|\psi(n, m, 0), \psi(n, m, 1)|_{k_{1}=0}, \quad g=|\psi(n, m,-1), \psi(n, m, 1)|_{k_{1}=0},
$$

where the basic vector column $\psi(n, m, l)$ is (3.16) with even generator (3.15), $N=2$ and $l_{0}=2$, which is the same as that we used to generate rational solution (3.17) for H3.

\subsection{For bilinearization-II}

The second bilinearlization for Q1 is derived through the transformation [6]

$$
u=A \alpha^{n} \beta^{m} \frac{\overline{\bar{f}}}{f}+B \alpha^{-n} \beta^{-m} \frac{\frac{f}{f}}{f}, \quad A B=\delta^{2} r^{2} / 16,
$$

with the parametrization is

$$
-\frac{1}{4} r(1-\alpha)^{2} / \alpha=p, \quad-\frac{1}{4} r(1-\beta)^{2} / \beta=q .
$$

The bilinear form is exactly the same as (3.1), which is a bilinear H3. In this case Q1 can share Theorem 1 and the Casoratian column vector $\psi$ with H3, as given in Section 3. By the same Casoratians $f$ that we used to generate solutions (3.14) and (3.17) in Section 3, the corresponding solutions of Q1 are

$$
\begin{aligned}
u_{n, m}= & A \alpha^{n} \beta^{m} \frac{\left(c-k_{1}\right)^{4}-\left[\left(c^{2}-k_{1}^{2}\right) D_{1}+8 k_{1} c\right]\left(c^{2}-k_{1}^{2}\right) Q_{n, m}-\left(c+k_{1}\right)^{4} Q_{n, m}^{2}}{1-D_{1} Q_{n, m}-Q_{n, m}^{2}} \\
& +B \alpha^{-n} \beta^{-m} \frac{\left(c-k_{1}\right)^{-4}-\left[\left(c^{2}-k_{1}^{2}\right) D_{1}-8 k_{1} c\right]\left(c^{2}-k_{1}^{2}\right)^{-3} Q_{n, m}-\left(c+k_{1}\right)^{-4} Q_{n, m}^{2}}{1-D_{1} Q_{n, m}-Q_{n, m}^{2}},
\end{aligned}
$$

and

$$
\begin{aligned}
u_{n, m}= & A \alpha^{n} \beta^{m} c^{4}\left(1+\frac{2 a b}{c(b n+a m)+2 a b}\right) \\
& +B \alpha^{-n} \beta^{-m} c^{-4}\left(1-\frac{2 a b}{c(b n+a m)+2 a b}\right) .
\end{aligned}
$$

where $A B=\delta^{2} r^{2} / 16, Q_{n, m}$ and $D_{1}$ are respectively defined in (3.14b) and (3.14c). (4.5) gives a rational solution of Q1. 


\section{Conclusions}

We have shown that H3 and Q1 models in the ABS's list admit more solutions than solitons. The coefficient(or transform) matrix $A_{[\mu]}$ in the Casoratian conditions, i.e., those difference equation sets for the Casoratian column vector $\psi$, plays an important role. When $A_{[\mu]}$ is a diagonal matrix with $N$ distinct eigenvalues, we get $N$-soliton solutions. $A_{[\mu]}$ can also be a LTTM with $N$ same eigenvalues. In this case, one gets solutions different from solitons. Particularly, $k_{1}=0$ generates rational solutions. $A_{[\mu]}$ can also be a combination of diagonal blocks and LTTM blocks. We note that in continuous cases these LTTM-type solutions (related to LTTMs) can be considered as limit solutions of solitons (cf. [11]), and this is also true for discrete cases. H1 admits LTTMtype solutions [12] but it does not have rational solutions. The reason is that an invertible $A_{[\mu]}$ is needed in the Casoratian proof for $\mathrm{H} 1$, but this will be broken if taking $k_{1}=0$. $\mathrm{H} 2$ has the same situation as H1. In fact, for $\mathrm{H} 1$ and H2, the basic Casoratian entry is

$$
\psi_{i}(n, m, l)=\rho_{i}^{+}\left(a+k_{i}\right)^{n}\left(b+k_{i}\right)^{m} k_{i}^{l}+\rho_{i}^{-}\left(a-k_{i}\right)^{n}\left(b-k_{i}\right)^{m}\left(-k_{i}\right)^{l},
$$

i.e., $c=0$ in (3.5) for H3, or $\delta=0$ in (4.3) for Q1. However, for H3 and Q1, it is just the existence of $c$ and $\delta$ to guarantee the non-triviality of $A_{[\mu]}$ when $k_{1}$ goes to zero. To keep $A_{[\mu]}$ invertible is also the criterion to examine rational solution reduction $(\delta=0)$ for H3 and Q1. Obviously, the rational solutions derived from the first bilinear form of Q1 do not admit reduction of $\delta=0$, but others do. For $\mathrm{H} 1$ and $\mathrm{H} 2$, trying to introduce an auxiliary parameter (for example, $c$ for $\mathrm{H} 3$ ) in their Casoratian entry so that the transform matrix $A_{[\mu]}$ is still invertible as $k_{1} \rightarrow 0$ might be a possible way to get their rational solutions in Caosratian form. This is left for further discussion.

\section{Acknowledgements}

The authors are very grateful to the referees for their invaluable comments. This project is supported by the NSF of China (11071157) and Shanghai Leading Academic Discipline Project (No. J50101).

\section{References}

[1] Nijhoff F.W., Walker A.J., The discrete and continuous Painlevé VI hierarchy and the Garnier systems, Glasgow Math. J. 43A (2001), 109-123, nlin.SI/0001054.

[2] Adler V.E., Bobenko A.I., Suris Yu.B., Classification of integrable equations on quad-graphs. The consistency approach, Comm. Math. Phys. 233 (2003), 513-543, nlin.SI/0202024.

[3] Atkinson J., Hietarinta J., Nijhoff F., Seed and soliton solutions of Adler's lattice equation, J. Phys. A: Math. Theor. 40 (2007), F1-F8, nlin.SI/0609044.

[4] Atkinson J., Hietarinta J., Nijhoff F., Soliton solutions for Q3, J. Phys. A: Math. Theor. 41 (2008), 142001, 11 pages, arXiv:0801.0806.

[5] Nijhoff F., Atkinson J., Hietarinta J., Soliton solutions for ABS lattice equations. I. Cauchy matrix approach, J. Phys. A: Math. Theor. 42 (2009), 404005, 34 pages, arXiv:0902.4873.

[6] Hietarinta J., Zhang D.J., Soliton solutions for ABS lattice equations. II. Casoratians and bilinearization, J. Phys. A: Math. Theor. 42 (2009), 404006, 30 pages, arXiv:0903.1717.

[7] Hietarinta J., Zhang D.J., Multisoliton solutions to the lattice Boussinesq equation, J. Math. Phys. 51 (2010), 033505, 12 pages, arXiv:0906.3955.

[8] Atkinson J., Nijhoff F., A constructive approach to the soliton solutions of integrable quadrilateral lattice equations, Comm. Math. Phys. 299 (2010), 283-304, arXiv:0911.0458.

[9] Nijhoff F., Atkinson J., Elliptic $N$-soliton solutions of ABS lattice equations, Int. Math. Res. Not. 2010 (2010), no. 20, 3837-3895, arXiv:0911.0461. 
[10] Ablowitz M.J., Satsuma J., Solitons and rational solutions of nonlinear evolution equations, J. Math. Phys. 19 (1978), 2180-2186.

[11] Zhang D.J., Notes on solutions in Wronskian form to soliton equations: KdV-type, nlin.SI/0603008.

[12] Zhang D.J., Hietarinta J., Generalized solutions for the H1 model in ABS list of lattice equations, in Nonlinear and Modern Mathematical Physics (July 15-21, 2009, Beijing), Editors W.X. Ma, X.B. Hu and Q.P. Liu, AIP Conf. Proc., Vol. 1212, Amer. Inst. Phys., Melville, NY, 2010, 154-161.

[13] Freeman N.C., Nimmo J.J.C., Soliton solutions of the Korteweg-de Vries and Kadomtsev-Petviashvili equations: the Wronskian technique, Phys. Lett. A 95 (1983), 1-3. 